\title{
EL FALLO DEL 18 DE DICIEMBRE DE 2003 REFERENTE A LA SOLICITUD DE REVISIÓN DEL FALLO DEL 11 DE SEPTIEMBRE DE 1992, EN EL CASO DE LA DISPUTA FRONTERIZA TERRESTRE, INSULAR Y MARÍTIMA (EL SALVADOR/HONDURAS: NICARAGUA INTERVINIENDO) (El SALVAdOR VS. HONDURAS)
}

Tania PaCheco Blandino

Abogada y Notario Público.

\section{Introducción}

$\mathrm{E}$ 1 presente documento no pretende ser un estudio pormenorizado de todos los aspectos relativos al fallo de 18 de diciembre de 2003 de la Corte Internacional de Justicia ${ }^{1}$ respecto a la solicitud presentada por la República de El Salvador ${ }^{2}$ el 10 de septiembre de 2002; tan sólo contiene los puntos esenciales de dicho caso y unas cuantas conclusiones que se pueden derivar del mismo.

Su importancia para la región radica en constituir la solución definitiva de una disputa territorial que hubiera podido llegar a constituir un nuevo factor de desestabilización en la zona ${ }^{3}$, es además, una muestra más del espíritu pacifista de los Estados centroamericanos, que confiando cada vez con mayor convicción en los métodos internacionales de resolución de conflicto, depositan sus disputas ante el máximo tribunal de Naciones Unidas, a fin de encontrar una salida viable a su disputa.

En la historia de la Corte, únicamente tres veces se ha introducido solicitud de revisión de un fallo ${ }^{4}$ y en todos los casos se ha decidido que dichas solicitudes eran improcedentes.

La revisión de un fallo, al igual que la intervención de un tercer Estado en un caso, continúa siendo terreno inexplorado y sinuoso para todo Estado que se aventura en dicha empresa.

\footnotetext{
1 En adelante: la Corte.

2 En adelante: El Salvador.

3 Honduras ante los problemas que en la ejecución del fallo de 1992, comenzó a advertir sobre la posibilidad de pedir al Consejo de Seguridad de Naciones Unidas que obligara a El Salvador a cumplir con el fallo. Véase "Honduras pedirá a la ONU garantizar fallo de La Haya" La Prensa, 12 de enero de 2003, nacionales.

4 Solicitud de Revisión del Fallo del 11 de septiembre de 1992, en el caso de la disputa Fronteriza terrestre, insular y marítima (El Salvador/Honduras: Nicaragua Interviniendo) (El Salvador vs. Honduras), Solicitud de Revisión e interpretación del Fallo de 24 de febrero de 1982 en el caso referente a la Plataforma Continental (Túnez/La Jamahiriya Árabe Libia) (Túnez vs. La Jamahiriya Árabe Libia) y Solicitud de Revisión del fallo de 11 de julio de 1996 en el caso concerniente a la Aplicación de la Convención sobre la Prevención y Castigo del delito de Genocidio (Bosnia y Herzegobina vs. Yugoslavia), Excepciones Preliminares (Yugoslavia vs. Bosnia y Herzegobina).
} 


\section{El Artículo 61 del Estatuto ${ }^{5}$ de la Corte Internacional de Justicia}

La base jurídica de toda solicitud de revisión está dada por el artículo 61 del Estatuto y los artículos 99 y 100 del Reglamento de la Corte Internacional de Justicia ${ }^{6}$.

El artículo 61 del Estatuto establece:

1. Sólo podrá pedirse la revisión de un fallo cuando la solicitud se funde en el descubrimiento de un hecho de tal naturaleza que pueda ser factor decisivo y que, al pronunciarse el fallo, fuera desconocido de la Corte y de la parte que pida la revisión, siempre que su desconocimiento no se deba a negligencia.

2. La Corte abrirá el proceso de revisión mediante una resolución en que se haga constar expresamente la existencia del hecho nuevo, en que se reconozca que éste por su naturaleza justifica la revisión, y en que se declare que hay lugar a la solicitud.

3. Antes de iniciar el proceso de revisión la Corte podrá exigir que se cumpla lo dispuesto por el fallo.

4. La solicitud de revisión deberá formularse dentro del término de seis meses después de descubierto el hecho nuevo.

5. No podrá pedirse la revisión una vez transcurrido el término de diez años desde la fecha del fallo."”

Según el artículo antes citado el proceso de revisión se abre con un fallo de la Corte declarando la solicitud admisible por el motivo en éste previsto. El artículo $99^{8}$ del Reglamento prevé expresamente un proceso sobre el fondo del asunto si, en su primer fallo, la Corte ha declarado la solicitud admisible.

Tanto el Estatuto como el Reglamento de la Corte, establecen un proceso de 2 fases:

a. Admisibilidad'.

1) Fondo de la Fase de la admisibilidad

2) Ejecución del fallo original

b. Fondo de la Revisión.

5 En adelante: el Estatuto.

${ }^{6}$ En adelante: el Reglamento.

7 Traducción de la Corte, disponible en página web: http://www.icj-cij.org.

8 Artículo 99, "1. Una solicitud de revisión de un fallo se introduce mediante un escrito que debe de contener las indicaciones necesarias que muestre que las condiciones previstas en el artículo 61 del Estatuto fueron llenadas. 2. La parte adversa tiene el derecho de presentar las observaciones escritas sobre la admisibilidad de la solicitud dentro del término fijado por la Corte o, por el Presidente de la Corte, en caso de que la Corte no estuviera en sesión. 3. La Corte, antes de dar su fallo sobre la admisibilidad de la solicitud puede conceder a las partes la posibilidad de presentar sus puntos de vista al respecto. 4. Si la solicitud se declara admisible, la Corte fijará, después de haber escuchado el punto de vista de las partes, los plazos para todo el procedimiento ulterior que estime necesario sobre el fondo de la demanda. 5. Si la Corte decide subordinar la admisión de la solicitud de revisión a la ejecución anterior del fallo, debe de emitir una orden en este sentido."

9 Véase, solicitud de Revisión e interpretación del Fallo de 24 de febrero de 1982 en el caso referente a la Plataforma Continental (Túnez/ La Jamahiriya Árabe Libia) (Túnez v. La Jamahiriya Árabe Libia), Fallo, CIJ, 1985 p. 192, párras. 8 y 10. Solicitud de Revisión del fallo de 11 de julio de 1996 en el caso concerniente a la Aplicación de la Convención sobre la Prevención y Castigo del delito de Genocidio (Bosnia y Herzegobina vs. Yugoslavia), Excepciones Preliminares (Yugoslavia vs. Bosnia y Herzegobina), Fallo de 13 de febrero de 2003, párr. 15. 


\section{a. Admisibilidad}

\section{1) Fondo de la fase de la admisibilidad}

En la fase de admisibilidad se debe constatar que la parte solicitante de la revisión haya satisfecho cada uno de los requisitos estipulados en el artículo 61 antes citado. Se examina uno por uno los requisitos, con uno que no sea cumplido la Corte procede a dejar de estudiar la solicitud y por ende, a pronunciar su fallo sobre la admisibilidad de la solicitud. ${ }^{10}$

En esta primera fase, en el caso objeto del presente estudio, previamente al estudio de la admisibilidad la Corte dictó una orden mediante la cual constituía la Sala que se encargaría de estudiar la solicitud de revisión. Dicha Sala se limitó a analizar si El Salvador en su solicitud había cumplido con las condiciones contempladas en el estatuto, que para efecto de nuestro trabajo dividiremos en dos límites o requisitos para su aceptación:

\section{1) Ratione facti novum ${ }^{11}$}

La solicitud debe estar basada en el "descubrimiento" de un "hecho", el cual debe de cumplir con una serie de requisitos. Debe ser de tal naturaleza, que constituya un "factor decisivo" para el caso en cuestión. Además tiene que ser un hecho "desconocido" tanto para la Corte, como para la parte que solicita la revisión, en el momento en que el fallo fue pronunciado. La ignorancia de este hecho no debe ser debido a "negligencia" de la parte que lo alega. Es decir, una vez aceptado que existe un hecho nuevo, se debe constatar "la ausencia de negligencia del demandante en el desconocimiento del hecho nuevo alegado y la naturaleza decisiva del mismo para alterar el fallo" 12 .

\section{2) Ratione temporis ${ }^{13}$}

Conocido también como doble límite en razón del tiempo, en cuando se debe de cumplir con dos requisitos.

\footnotetext{
${ }^{10}$ Véase, solicitud de Revisión e interpretación del Fallo de 24 de febrero de 1982 en el caso referente a la Plataforma Continental (Túnez/ La Jamahiriya Árabe Libia) (Túnez v. La Jamahiriya Árabe Libia), Fallo, CIJ, Reportes de 1985 p. 207, párr. 29. Solicitud de Revisión del fallo de 11 de julio de 1996 en el caso concerniente a la Aplicación de la Convención sobre la Prevención y Castigo del delito de Genocidio (Bosnia y Herzegobina vs. Yugoslavia), Excepciones Preliminares (Yugoslavia vs. Bosnia y Herzegobina), Fallo de 13 de febrero de 2003, párras. 72 y 73 .

${ }^{11}$ VERZIJL, JHM. The jurisprudence of the World Court. Vol. I, 1965, págs.349-350.

${ }^{12}$ DIEZ-HOCHLEITNER; (REMIRO BROTONS-Director), Derecho Internacional, 1997, pág. 882.

${ }^{13}$ ROSENNE, Shabtai. The law and practice of the International Court, 1985, pág. 429.
} 
La solicitud que contempla el hecho en cuestión debe ser presentada dentro del término de 6 meses del descubrimiento del nuevo hecho ${ }^{14} \mathrm{y}$ antes de la expiración del término de 10 años desde la fecha del pronunciamiento del fallo.

Según la Corte cada uno de los requisitos previstos debe de ser satisfecho. ${ }^{15}$

\section{2) Ejecución previa del Fallo}

Según lo establecido en el artículo 61 del Estatuto inciso 3 y el artículo 99 del Reglamento, inciso 5, antes de iniciar el proceso de revisión la Corte puede exigir la ejecución del fallo que se pretende revisar.

Esto se hace a efecto de no perjudicar la res judicata o cosa juzgada, es decir, para que no sea utilizada una solicitud de revisión para dar largas o incumplir la ejecución de un fallo. ${ }^{16}$

3. Admisibilidad en el caso referente a la solicitud de revisión del fallo del 11 de septiembre de 1992, en el caso de "La disputa fronteriza terrestre, insular y marítima (El Salvador/Honduras: Nicaragua interviniendo)" (El Salvador vs. Honduras)

\section{a. El Procedimiento}

El 10 de septiembre de 2002 El Salvador presentó ante la Corte una solicitud mediante la cual, con base en el artículo 61 del Estatuto de la Corte y los artículos 99 y 100 del Reglamento de la Corte, sometía ante ésta para su revisión el fallo de 11 de septiembre de 1992 emitido por una Sala ad hoc de la Corte, creada para resolver el caso referente a "La disputa fronteriza, terrestre, insular y marítima (El Salvador/Honduras: Nicaragua interviniendo)"17. Este mismo día, según lo establecido en el artículo 40, párrafo 2 del Estatuto, el Secretario General de la Corte trasladó copia certificada de la demanda a la República de Honduras ${ }^{18}$. De

\footnotetext{
${ }^{14}$ Valga aclarar que no todo hecho posterior a un fallo puede ser considerado como nuevo. Cf. Solicitud de Revisión del fallo de 11 de julio de 1996 en el caso concerniente a la Aplicación de la Convención sobre la Prevención y Castigo del delito de Genocidio (Bosnia y Herzegobina vs. Yugoslavia), Excepciones Preliminares (Yugoslavia vs. Bosnia y Herzegobina), Fallo de 13 de febrero de 2003, párras. 67. "Un hecho que ocurre muchos años después de que un fallo fue emitido no constituye un 'nuevo' hecho para los efectos del artículo 61; al igual que las consecuencias legales que dicho hecho pueda tener."

${ }^{15}$ Solicitud de Revisión del fallo de 11 de julio de 1996 en el caso concerniente a la Aplicación de la Convención sobre la Prevención y Castigo del delito de Genocidio (Bosnia y Herzegobina vs. Yugoslavia), Excepciones Preliminares (Yugoslavia vs. Bosnia y Herzegobina), Fallo de 13 de febrero de 2003, párr. 17.

${ }^{16}$ GUYOMAR, Geneviève. Commentaire du Règlement de la Court Internationale de Justisce, 1983, pág. 628. "une partie...ne retardât...l'exécution d'un arrêt de la Cour sous prétexte que dans ce délai un fait nouveau pouvait éter découvert qui entraînât le retrait". C.P.J.I. Comité de juristes, págs. 744 et s.

${ }^{17}$ ICJ Reports 1992, p.351.

${ }^{18}$ En adelante: Honduras.
} 
igual forma hizo llegar copia a la República de Nicaragua, a fin de que estuviera informada sobre el caso, ya que, de acuerdo a lo establecido en el artículo 62 del Estatuto, había sido autorizada a intervenir en el caso original. ${ }^{19}$

En su escrito, El Salvador, según lo establecido en el artículo 100 párrafo 1 del Reglamento $^{20}$, pedía a la Corte constituir una Sala cuyo objetivo sería resolver la demanda de revisión de la sentencia de 11 de septiembre de 1992, dicha Sala debía ser constituida respetando los términos acordados entre El Salvador y Honduras en el Acuerdo Especial de 24 de mayo de $1986^{21}$.

Las partes, previamente consultadas por el Presidente de la Corte (6 de noviembre de 2002), expresaron su deseo de constituir una nueva Sala con 5 miembros, de los cuales 2 serían los jueces ad hoc de cada una de las partes, según lo establecido en el artículo 31, párrafo 3 del Estatuto. En comunicación fechada 7 de noviembre de 2002 El Salvador, informó a la Corte que su gobierno había designado a S. E. Don Felipe A. Paolillo como magistrado ad hoc. Honduras por su parte, en comunicación fechada 18 de noviembre de 2002, designó a Don Santiago Torres Bernández ${ }^{22}$. El 26 de noviembre de 2002 se eligió entre los miembros de la Corte, los tres magistrados que integrarían la Sala: Presidente Guillaume, magistrados Rezek y Buergenthal.

Por orden de 27 de noviembre de 2002, la Corte, de acuerdo a lo establecido en el artículo 26, párrafo 2 del Estatuto y el artículo 17 del Reglamento y lo solicitado por las partes, constituyó una Sala especial que se encargaría de resolver el caso.

Conforme al artículo 92, párrafo 2 y 99, párrafo 2 del Reglamento y orden antes mencionada, la Corte fija el 1 de abril de 2003 como fecha límite para la presentación de las Observaciones Escritas de Honduras relativas a la admisibilidad de

\footnotetext{
${ }^{19}$ Véase "Solicitud de intervención del Gobierno de Nicaragua" presentada en la Secretaría de la Corte el 17 de noviembre de 1989 y sentencia de 13 de septiembre de 1990, por la cual se le permite intervenir a Nicaragua "Caso concerniente a la disputa fronteriza, terrestre, insular y marítima (El Salvador/Honduras) solicitud de permiso de intervención.

${ }^{20}$ Artículo 100: "1. Si el fallo a ser interpretado o revisado fue emitido por la Corte, ésta será quien conocerá de la solicitud de interpretación o revisión. Si el fallo fue emitido por una sala, ésta conocerá de la solicitud de interpretación o revisión. 2. La decisión de la Corte o de la sala sobre la solicitud de interpretación o revisión del fallo constituirá nuevo fallo."

${ }^{21}$ Véase texto en español en ICJ Reports 1990, p. 95. El artículo 1 que regulaba lo referente a la constitución de la sala establece: “...las Partes someten las cuestiones mencionadas en el Artículo Segundo del presente Compromiso a una Sala de la Corte Internacional de Justicia, compuesta por tres miembros, con la anuencia de las Partes, las cuales la expresarán en forma conjunta al Presidente de la Corte, siendo esta conformidad esencial para la integración de la Sala, que se constituirá de acuerdo a los Procedimientos establecidos en el Estatuto de la Corte y en el presente Compromiso. 2. Adicionalmente, integrarán la Sala dos jueces ad-hoc especialmente nombrados uno por El Salvador y otro por Honduras; los que podrán tener la nacionalidad de las Partes."

${ }^{22}$ Quien en el caso original, tras el fallecimiento del Juez Ad hoc Virally, fue designado por Honduras para sustituirlo. Cf. Land, Island and Maritime Frontier Dispute (El Salvador/Honduras: Nicaragua intervening), Judgment of 11 September 1992, ICJ Reports 1992, párras. 6 y 8 en pág. 359.
} 
la solicitud, reservando el establecimiento de los plazos siguientes del proceso a decisiones posteriores. Honduras presentó sus Observaciones Escritas en la fecha fijada.

El Salvador, por su parte, en comunicación fechada 8 de abril de 2003, señalaba que Honduras en sus Observaciones Escritas había presentado nuevos documentos con sus correspondientes argumentos, los cuales requerían una correspondiente contestación por parte de El Salvador, acompañada de los documentos necesarios. Honduras en comunicación fechada 24 de abril de 2003 se opone a dicha solicitud.

La Sala decidió, después de haberse reunido el Presidente de la misma con los agentes de las partes, que la presentación de alegatos escritos adicionales no sería necesaria. Pero si El Salvador deseaba presentar nuevos documentos, dicha solicitud sería considerada de acuerdo con el procedimiento establecido en el artículo 56 del Reglamento. Dicha decisión fue notificada a las partes en comunicación de fecha 8 de mayo de 2003.

Mediante comunicación de 23 de junio de 2003, El Salvador pidió autorización para para presentar nuevos documentos (de acuerdo al artículo 56 del Reglamento), los cuales habiendo sido presentados ante la Secretaría General fueron transmitidos a Honduras (de conformidad con el artículo 56 párrafo 1 del Reglamento). Honduras, en comunicación de 10 de julio de 2003 informó a la Sala que objetaba la presentación de dichos documentos. Ambos países fueron autorizados a presentar sus observaciones respecto a este punto, lo que hicieron mediante comunicaciones fechadas 17 (El Salvador) y 24 de julio de 2003 (Honduras). Una vez examinado lo expresado por las partes, la Sala decidió, de acuerdo con el artículo 56, párrafo 2 del Reglamento, autorizar la presentación de sólo parte de los documentos presentados por El Salvador. La Sala además decidió no autorizar la presentación de un nuevo documento adjunto a las Observaciones de Honduras.

En comunicación de 19 de julio de 2003 se le notificó a las partes lo decidido y de acuerdo al artículo 56, párrafo 3, Honduras fue autorizada a presentar los comentarios que estimara pertinentes (a mas tardar el 19 de agosto de 2003) sobre los documentos que la Sala había autorizado presentar a El Salvador, debiendo también presentar los documentos respaldo de sus comentarios.

El 19 de agosto de 2003, dentro del plazo establecido, Honduras presentó sus comentarios ante la Secretaría junto con 4 documentos soporte.

Finalmente, las audiencias públicas tuvieron lugar el 8, 9, 10 y 12 de septiembre de 2003. Y el fallo fue pronunciado el 18 de diciembre de 2003. 


\section{b. El Objeto de la Solicitud}

Mediante fallo de 11 de septiembre de 1992 la Sala de la Corte integrada para resolver el caso referente a "La Disputa fronteriza terrestre, insular y marítima (El Salvador/Honduras: Nicaragua interviniendo)", decidió el curso de la frontera terrestre entre El Salvador y Honduras en los seis sectores en disputa. En el mismo fallo la Sala resolvió la disputa entre las partes sobre el estatus legal de las aguas en el Golfo de Fonseca y fuera de éste.

El Salvador presentó una solicitud ante la Corte a fin de revisar la sentencia de 1992 únicamente en lo relativo al sexto sector de la frontera terrestre, que se extiende entre Los Amates y el Golfo de Fonseca.

Durante el caso original Honduras alegaba que en ese sector "la frontera ... sigue el actual curso [del río Goascorán] desembocando en el Golfo al noroeste de la Islas Ramaditas en la Bahía de la Unión.” El Salvador, por su parte, pedía que la frontera fuera definida por "el curso previo seguido por el río... y que dicho curso, abandonado, podía ser trazado y alcanzaría el Golfo en el Estero La Cutú." 23 La Sala finalmente decidió por unanimidad acoger la petición de Honduras ${ }^{24}$.

\section{Lo Solicitado}

En su solicitud El Salvador pedía a la Corte:

a. Proceder a formar una Sala que se encargara de resolver la solicitud de revisión del fallo, teniendo en cuenta los términos acordados por El Salvador y Honduras en el Acuerdo Especial de 24 de mayo de 1986.

b. Declarar su solicitud admisible, debido a la existencia de nuevos hechos cuya naturaleza ocasionara que el caso se abriera a revisión bajo lo estipulado en el artículo 61 del Estatuto.

c. Una vez que la solicitud fuera admitida, proceder a la revisión del fallo de 11 de septiembre de 1992 y que un nuevo fallo determinara la línea fronteriza en el sector sexto de la frontera terrestre entre El Salvador y Honduras de la siguiente forma:

"comenzando desde la antigua desembocadura del Río Goascorán en el sector conocido como El Estuario La Cutú, situado $13^{\circ} 22^{\prime} 00^{\prime \prime} \mathrm{N}$ y longitud $87^{\circ} 41^{\prime} 25^{\prime \prime} \mathrm{O}$, la frontera sigue el viejo curso del río Goascorán por una distancia de 17,300 metros hasta el lugar conocido como el Rompición de Los Amates situado en latitud

\footnotetext{
${ }^{23}$ Land, Island and Maritime Frontier Dispute (El Salvador/Honduras: Nicaragua intervening), Judgment of 11 September 1992, ICJ Reports 1992. p. 359 párras. 306 en pág. 544.

${ }^{24}$ Ibid, párras. 321, 322 y 430 en págs. 552, 553 y 615.
} 
$13^{\circ} 26^{\prime} 29^{\prime \prime N}$ y longitud $87^{\circ} 43^{\prime} 25^{\prime \prime} \mathrm{O}$, que es donde el río Goascorán cambia su curso." 25

Honduras, por su parte, solicitaba:

Declarar inadmisible la demanda de revisión presentada por El Salvador el 12 de septiembre de 2002.

Una vez creada la Sala, en los alegatos orales, ambas partes reafirmaron su solicitud.

\section{Límite Ratione Facti: Nuevos Hechos}

\section{C.1 El Fallo de 1992 y El Sexto Sector Fronterizo}

Para poder comprender el caso en cuestión tendremos que abordar los puntos torales que fueron tratados en el caso original respecto al sexto sector de la frontera terrestre, de importancia en la solicitud de revisión.

En el caso original El Salvador reconocía que el río Goascorán había sido adoptado como frontera provincial durante la época de la colonización española. Según El Salvador el río Goascorán en cierto momento había cambiado su curso a la posición que tiene actualmente y a partir de este hecho, su argumento jurídico, se centraba en que la frontera constituida por el curso de un río, cuando dicho río súbitamente cambia su curso y forma uno nuevo, este proceso de avulsión, no debía de cambiar el límite fronterizo fijado, que debía continuar siendo el curso original del río.

Tal era la regla tanto en derecho colonial español como en derecho internacional. Por lo cual, de acuerdo con El Salvador, la frontera entre los dos Estados debía ser establecida no a lo largo del curso actual del río que desemboca en la Bahía de la Unión, sino a lo largo del curso anterior que fuera abandonado por el río, probablemente en el siglo XVII, que desembocaba en El Estero La Cutú.

La Sala en su fallo de 1992 concluyó que no se le habían presentado pruebas de que tal cambio abrupto del curso del río hubiera ocurrido. Sin embargo la Sala no tomó partido en cuanto a las consecuencias que una avulsión, ocurrida antes o después de 1821, hubiera tenido respecto a las fronteras provinciales o las fronteras entre Estados, de acuerdo al derecho colonial español o al derecho internacional.

Por otro lado, un punto de vital importancia para la actual Sala, fue el hecho que la Sala en su fallo de 1992 había encontrado que cualquier afirmación de El

\footnotetext{
${ }^{25}$ Application for revision of the Judgment of 11 September 1992 in the case concerning the land, island and maritime frontier dispute (El Salvador/Honduras: Nicaragua intervening) (El Salvador v. Honduras), Judgment of 18 December 2003, pár. 12.
} 
Salvador que la frontera en el sector en cuestión seguía el antiguo curso abandonado del río debía ser rechazado por tratarse de una nueva pretensión incompatible con la historia del diferendo. En este sentido, para la Sala original, El Salvador en muchas ocasiones, incluyendo en particular en las negociaciones de Saco entre los dos Estados en 1880, había mostrado una conducta que excluía cualquier reclamo según el cual la frontera de 1821 no fuera el curso seguido por el río en ese momento.

La Sala, entonces, consideró los elementos de prueba que le fueron presentados referentes al curso del río Goascorán en 1821. Examinó en particular una "Carta Esférica" de El Gulfo de Fonseca preparado por el capitán y navegantes de la expedición El Activo, que zarpó en 1794, bajo las instrucciones del Virrey de México, para llevar a cabo estudios hidrográficos en el Golfo. La Sala encontró que la "Carta" era inconsistente con el antiguo curso del río, alegado por El Salvador y, además, con cualquier otro curso que no fuera el actual.

La Sala concluyó que el reporte de la expedición de 1794 y la "Carta Esférica" dejaban poco lugar a duda de que el río Goascorán en 1821 siguiera en ese entonces el actual curso del río.

\section{C.2 Los Nuevos Hechos}

El Salvador presentó documentos, para respaldar un hecho que ya había alegado durante el caso original y que la Sala en cuestión había desestimado. Para efectos del presente estudio, se dividirán en los documentos que sustentan este hecho y el hecho en sí de la avulsión del Goascorán.

Aunque el hecho siempre fuera la avulsión del río Goascorán, El Salvador se valió del descubrimiento de versiones diferentes de documentos presentados por Honduras en el caso original (La Carta Esférica y el Reporte de 1794), la presentación de nuevos reporte y estudio realizados en 2002 que debido a la ausencia de tecnología no había podido ser presentado en el caso original y varias publicaciones a las cuales no había podido tener acceso anteriormente por la situación en que se encontraba el país.

\section{C.2.1 Documentos que demuestran la avulsión del Goascorán}

El primer punto a demostrar era, si el descubrimiento de un documento podía ser considerado como un hecho motivador de una revisión.

El Salvador, respaldado, entre otros documentos, en el Reporte de 5 de agosto de 2002: “Aspectos geológicos, hidrológicos e históricos del delta del Goascorán”, afir- 
maba poseer evidencias científicas, técnicas e históricas que demostrarían, contrario a lo decidido por la Sala, que el Goascorán cambió su curso y que dicho cambio abrupto había probablemente ocurrido como resultado de un ciclón en 1792.

En particular señalaba que los estudios científicos y técnicos que para efectos de esta revisión había presentado, no podían haber sido llevados a cabo previamente, dado el estado de la ciencia y la tecnología en 1992 y la situación política prevaleciente en esa época en el sexto sector de la frontera y, generalmente, en El Salvador y en la región. Respecto a ciertas publicaciones ${ }^{26}$ en respaldo de su teoría, El Salvador expresó que no había podido tener acceso a los documentos del Archivo Nacional de Honduras, a pesar de sus esfuerzos y que no había podido localizarlos en los archivos de otros Estados a los cuales tuvo acceso.

El Salvador señalaba que dichas pruebas constituían "nuevos hechos" (según lo establecido en el artículo 61 del Estatuto). En este sentido se apoyaba en los travaux préparatoires (trabajos preparatorios) de la disposición del Estatuto de la Corte Permanente de Justicia Internacional, en la cual el artículo 61 está basado, que confirma que un documento puede ser considerado como "un nuevo hecho". También invocaba un laudo emitido el 7 de agosto y 25 de septiembre de 1922 por el Tribunal arbitral mixto Franco-germano en el caso Hein et Chamant c. Etat allemand, que según el punto de vista de El Salvador, reconoce que los elementos de prueba pueden constituir un "hecho".

El descubrimiento en (la Ayer Collection de la Newberry Library en Chicago) de una nueva copia de la "Carta Esférica" y del reporte de la expedición de El Activo, constituían también nuevos hechos en cuanto a que la Sala de 1992 únicamente contó con copia de los documentos presentados por Honduras que habían sido obtenidos del Museo Naval de Madrid.

Según El Salvador la importancia de los documentos descubiertos en Chicago radica en:

1) Valor probatorio: la existencia de diferentes versiones de estos documentos, las divergencias entre ellos y los anacronismos que comparten, ponían en evidencia el valor probatorio que la Sala en 1992 les concedió. Siendo además que los documentos de Madrid no gozaban de estatus oficial y no se había certificado su originalidad.

2) Constitución del factum o una fuente de su conocimiento: venir a constituir evidencia útil para revocar un hecho establecido en un fallo.

${ }^{26}$ En particular se refería a: Geografía de Honduras de Ulises Meza Cáliz publicada en 1916 y Monografía del Departamento del Valle, preparada bajo la dirección de Bernardo Galindo y Galindo y publicada en 1934. 
El Salvador justificaba que el hecho en cuestión preexistiera antes del fallo de 1992, con que dicho hecho no fuera conocido al momento que el fallo había sido pronunciado. Por tanto, era un "nuevo hecho" para los efectos del artículo 61 del Estatuto. Y su importancia decisiva recaía en que pondría en evidencia la inconsistencia de los documentos descubiertos en el Museo Naval de Madrid sobre los cuales la Sala infirió consecuencias geográficas muy importantes.

Para El Salvador la ignorancia hasta 2002, por su parte, de la existencia de copias de los documentos del Activo en colecciones situadas en lugares inopinados, no podía ser calificada de "negligente".

Honduras negaba que la presentación de los documentos encontrados en Chicago pudiera ser caracterizado como un nuevo hecho, ya que tan solo eran nuevas copias de un documento que ya había sido presentado a la Sala original. Entre estas tres cartas no existían grandes discrepancias, tan solo diferencias insignificantes. Finalmente, señalaba que las tres cartas colocaban en la posición actual la desembocadura del río Goascorán, punto que sirvió de base al fallo de 1992.

Por otro lado, Honduras alegaba que los nuevos documentos presentados por El Salvador eran parte de una prestigiosa colección pública y habían sido incluidos en el catálogo de la Newberry Library al menos desde 1927. Por lo cual, El Salvador podía fácilmente conocer esos documentos, faltando a su diligencia en la búsqueda y presentación de ellos antes de 1992. Según Honduras no se podía justificar dicha negligencia con los conflictos internos que vivía El Salvador en dicho período porque éstos no impedía la búsqueda de documentos fuera del territorio nacional.

Honduras concluía señalando que las condiciones del artículo 61 del Estatuto no habían sido satisfechas y que la solicitud de revisión fundada en el descubrimiento de una nueva carta y reporte no podía ser admitida.

La Sala en 1992 expresó respecto a la "Carta Esférica" que la posición de la desembocadura era inconsistente con el antiguo curso del río, alegado por El Salvador, o cualquier otro que no fuera el actual y siendo que en la misma no mencionaba antigua desembocadura del río Goascorán, esto sugiere que en 1796, ya había, por considerable período de tiempo, desembocado en el Golfo, según lo indicado en la Carta.

De igual forma la Sala en 1992 analizó el Reporte de la expedición y observó que también localizaba la boca del río Goascorán en su actual posición.

Con lo anterior la Sala en 1992 llegó a la conclusión de que el reporte de 1794 y la "Carta Esférica" dejaban poco lugar a duda de que el río Goascorán en 1821 corría donde se encuentra en la actualidad. 
Era claro, por tanto, que la Sala en su fallo de 1992 había sustentado lo dispuesto en éste, en cierta información proporcionada por la "Carta Esférica" y el reporte de la expedición El Activo, en su versión resguardada en Madrid. Teniendo la actual Sala que decidir si la Sala habría llegado a otra conclusión en 1992, si hubiera tenido acceso a los documentos encontrados en Chicago.

La Sala en 2003 concluyó que las nuevas cartas presentadas por El Salvador muestran El Estero La Cutú y la desembocadura del Río Goascorán en la misma posición de la versión de Madrid, es decir, en su actual localización. Por tanto, la nueva carta presentada por El Salvador no contradice las conclusiones alcanzadas por la Sala en 1992, por el contrario, sirve para confirmarles.

Respecto a la nueva versión del reporte de la expedición de El Activo encontrada en Chicago, difiere de la versión de Madrid únicamente en ciertos detalles tales como ortografía, acentuación y leyendas de las mismas. El cuerpo del documento es el mismo, en particular, en lo referente a la identificación de la desembocadura del Goascorán. De ahí que los nuevos documentos presentados por El Salvador confirmaran las conclusiones alcanzadas por la Sala en 1992.

Por todo lo anterior la Sala en 2003 concluyó que los denominados por El Salvador nuevos hechos, en relación con los documentos como la "Carta Esférica" y el reporte de la expedición de El Activo no constituían factores decisivos respecto al fallo en cuestión. ${ }^{27}$

\subsection{La Avulsión del Goascorán}

El Salvador sostenía que las pruebas que estaba ofreciendo establecían la existencia de un antiguo lecho del Goascorán que desembocaba en el Estero La Cutú y la avulsión del río a mediados del siglo XVIII, o al menos el considerar dicha avulsión como plausible.

Según El Salvador el fallo de 1992 está fundado en el rechazo de una avulsión que, según el punto de vista de la Sala, no había sido probada y que dicha avulsión en estos momentos dejaba de ser conjeturas para tornarse en un hecho probado que se produjo realmente. De acuerdo al derecho colonial español, las fronteras de las provincias pertenecían invariables, a pesar de la avulsión, hasta 1821. El Salvador concluía que, contrario a lo que la Sala sostuvo en 1992, la frontera derivada del uti possidetis juris debería seguir dicho límite y no el nuevo curso del Goascorán.

\footnotetext{
${ }^{27}$ Application for revision of the Judgment of 11 September 1992 in the case concerning the land, island and maritime frontier dispute (El Salvador/Honduras: Nicaragua intervening) (El Salvador v. Honduras), Judgment of 18 December 2003, pár. 55.
} 
El Salvador señalaba que dadas todas las circunstancias del caso, en particular "la violenta guerra civil" que se vivía en El Salvador durante todo el período entre 1980 y el pronunciamiento del fallo de 11 de septiembre de 1992, la ignorancia de estos nuevos hechos referentes al curso del río Goascorán no se debía a su negligencia.

Por lo cual para El Salvador las condiciones fijadas por el artículo 61 del Estatuto estaban satisfechas, la solicitud de revisión fundada en la avulsión del río Goascorán debía ser admisible.

Honduras por su parte alegaba que respecto a la aplicación del artículo 61 del Estatuto, es "una jurisprudencia bien establecida que hay una distinción de naturaleza entre los hechos alegados y las pruebas presentadas para verificar un hecho real y que sólo, el descubrimiento de los primeros da lugar al derecho de revisión" 28 . En este respecto citaban la Opinión Consultivas dada el 4 de septiembre de 1924 por la Corte Permanente de Justicia Internacional referente al caso del Monasterio de Saint-Naoum. De acuerdo a Honduras, un "hecho" no puede ser "elementos de prueba presentados en apoyo de un argumento, una afirmación o un alegato." De ahí que, los elementos de prueba presentados por El Salvador no pudieran darle derecho a una revisión y no habiendo demostrado la existencia del nuevo hecho que "establezca que el Río Goascorán seguía el antiguo lecho que desembocaba en el Estero La Cutú o que un proceso de 'avulsión' ocurrido, o que ocurrió en una fecha dada" lo que estaba intentando era que la Sala realizara "una nueva interpretación de hechos previamente conocidos" y pedir una "verdadera reforma" ${ }^{29}$ del fallo de 1992.

Honduras señalaba que los hechos presentados por El Salvador, aún asumiendo que fueran nuevos y establecidos, no eran de tal naturaleza que ejercieran una influencia decisiva respecto al fallo de 1992. Asimismo, los materiales presentados por El Salvador a este respecto eran irrelevantes para la determinación hecha por la Sala. Esa decisión había sido basada únicamente en la constatación que "a partir de 1880, durante la negociación Saco, hasta 1972 El Salvador había considerado la frontera como siguiendo el curso del río tal cual era en 1821"30 considerándolo como una nueva pretensión, inconsistente con la historia previa de la disputa. No consideraba, por tanto, de importancia si había o no ocurrido una avulsión, la cual calificaba como irrelevante para la ratio decidendi de la Sala.

En todo caso para Honduras, El Salvador al ignorar en 1992 los hechos que pretendía presentar para sustentar la teoría de la avulsión, había incurrido en

${ }^{28}$ Ibid, pár. 31.

${ }^{29}$ Ibid.

${ }^{30}$ Ibid, párras. 32 . 
negligencia, ya que no probó que hubiera agotado los medios que le hubiera permitido tener un conocimiento diligente de los hechos que estaba alegando.

Honduras concluye que las condiciones establecidas en el artículo 61 del Estatuto no habían sido satisfechas, la solicitud de revisión fundada en la avulsión del río Goascorán inadmisible.

Respecto a lo expuesto por El Salvador la Sala recordó que la solicitud de revisión es admisible sólo si cada una de las condiciones estipuladas en el artículo 61 es satisfecha, si alguna de ellas falta, la solicitud debía de ser rechazada. La Sala comenzó analizando si los hechos alegados, supuestamente como nuevos hechos, son de tal naturaleza que constituyan un factor decisivo respecto a la sentencia de 1992.

La Sala al tratar este punto recordó los principios considerados por la Sala que originalmente lidiara con el caso, en la referente a la disputa de los dos Estados en los seis sectores terrestres.

Según la Sala, la frontera debía ser determinada mediante:

1. La aplicación de los principios generalmente aceptados en la América española de el uti possidetis juris, en virtud del cual las fronteras debían responder a los límites administrativos coloniales.

2. La situación resultante de la aplicación del uti possidetis juris, podía ser modificada por una decisión de un juez o por un tratado.

3. Factores como la acquiesciencia o el reconocimiento podían operar, en situaciones en las que existiera una variación, o al menos una interpretación de la situación resultante del uti possidetis juris. ${ }^{31}$

Aplicando los principios antes señalados respecto al sexto sector la Sala concluyó, respecto a los siguientes puntos:

a) La Sucesión de la Alcaldía Mayor de Tegucigalpa:

Que habiendo observado que durante el período colonial el Goascorán constituía frontera entre dos divisiones administrativas de la Capitanía General de Guatemala: la provincia de San Miguel y la Alcaldía Mayor de Minas de Tegucigalpa, ambas partes coincidían en que El Salvador había sucedido en 1821 al territorio de la Provincia de San Miguel. Pero disentían sobre si la Alcaldía Mayor de Tegucigalpa había pasado a Honduras. La Sala decidió este punto a favor de Honduras.

b) El lecho anterior del Río Goascorán constituía la frontera del uti possedetis juris:

${ }^{31}$ Land, Island and Maritime Frontier Dispute (El Salvador/Honduras: Nicaragua intervening), Judgment of 11 September 1992, ICJ Reports 1992. p. 359 párras. 28 y 67, págs. 380 y 401. 
Esta pretensión salvadoreña, según la Sala, estaba subordinada a la afirmación de si antiguamente el Goascorán corría a lo largo de ese lecho y a partir de un momento dado abruptamente cambió su curso a la posición actual. Dicho proceso de avulsión, según El Salvador, no constituía cambio de la frontera, que continuaría siendo el antiguo canal del río.

En este punto, la Sala señaló que no tenía conocimiento de ningún documento que demostrara que dicho cambio de curso del río hubiese ocurrido pero que si se le hubiese demostrado un cambio radical del curso del río, se hubiera podido decir que había habido una avulsión. Ha sido en esencia éste el punto, que ha llevado a El Salvador arriesgarse en el sinuoso pantano de una revisión.

La Sala concluyó en 1992 diciendo que cualquier afirmación de El Salvador de que la frontera sigue el lecho antiguo abandonado por el río en algún momento antes de 1821, debía ser rechazado. Agregando que esto constituiría una nueva pretensión inconsistente con la historia previa de la disputa.

Fue, sin embargo, este segundo punto, lo que sirvió a Sala en 2003 para señalar que cuando la Sala en su fallo de 1992 rechazó la pretensión de El Salvador de que la frontera de 1821 no seguía el curso del río en esa fecha, lo hizo basándose en la conducta de ese Estado durante el siglo XIX.

En conclusión, la Sala en 1992 no consideró de importancia si hubo o no una avulsión del Goascorán. Aún cuando dicha avulsión hubiera sido probada y aún cuando sus consecuencias legales fueran las inferidas por El Salvador, tal constatación no permitiría poner en duda la decisión tomada por la Sala en 1992.

Los hechos presentados por El Salvador no fueron considerados por la Sala como "factores decisivos" en relación con el fallo del cual se estaba pidiendo su revisión. A la luz del fallo de 1992, la Sala no podía llegar a otra conclusión, independientemente de la posición tomada por las partes a lo largo del proceso. ${ }^{32}$

\section{Situación en contexto de los nuevos hechos}

Para El Salvador era necesario una apropiada ubicación de los nuevos hechos para poder entenderlos. Debiendo tomar en cuenta factores como la erupción del volcán Cosigüina y la aparición de los Farallones de Cosigüina, las negociaciones Saco entre 1880 y 1884 y las características del curso inferior del río Goascorán.

\footnotetext{
${ }^{32}$ Application for revision of the Judgment of 11 September 1992 in the case concerning the land, island and maritime frontier dispute (El Salvador/Honduras: Nicaragua intervening) (El Salvador v. Honduras), Judgment of 18 December 2003, pár. 40.
} 
Para Honduras la presentación de esta nueva serie de factores constituía tratar de volver una revisión en una apelación, lo que iba en contra de la autoridad de la cosa juzgada.

La Sala estuvo de acuerdo con lo alegado por El Salvador referente a la necesidad de situar en el contexto adecuado los hechos en cuestión, sin embargo, consideró que ninguno de los hechos alegados por El Salvador constituían nuevos hechos, desconocidos por la Corte o las partes en el caso original y por lo tanto decidió que la solicitud de revisión era inadmisible ${ }^{33}$.

\section{Límite ratione temporis}

Ambas partes estuvieron de acuerdo de que la solicitud de revisión fue presentada dentro del término de diez años establecido en el párrafo 5 del artículo 61 del Estatuto, específicamente, un día antes de expirar el plazo. Honduras mantenía, sin embargo que dicho proceder, el solicitante mostraba mala fe procesal. Lo cual era negado por El Salvador ${ }^{34}$. Fue muy difícil sostener que únicamente 6 meses antes de vencido el término de 10 años de pronunciado el fallo se hayan encontrado y procesado todos los documentos necesarios para la presentación de la revisión en cuestión. Sin embargo, la Sala no necesitó pronunciarse al respecto.

\section{Ejecución previa del fallo}

Honduras en comunicación fechada 29 de octubre de 2002 pidió al Presidente de la Corte subordinar la solicitud de revisión a la ejecución previa del fallo de 1992. Hecho que fue interpretado in limine litis por El Salvador como una admisión implícita de la solicitud. Por lo cual el 24 de julio de 2003 Honduras decide no solicitar formalmente la ejecución de la sentencia.

Cabe señalar que en este sentido la Sala concluyó que la conducta hondureña no podía ser interpretada como una aceptación implícita de la solicitud de revisión presentada por El Salvador y aún que Honduras hubiera presentado la ejecución previa del fallo, sin esperar la decisión de la Sala respecto a la admisibilidad de la solicitud de El Salvador, dicha petición no hubiera implicado reconocimiento de la admisibilidad de la solicitud. ${ }^{35}$

Es además importante señalar que la Sala concluyó que sin importar el punto de vista de las partes, es la Corte, en todo caso, quien debe decidir respecto a la admisibilidad de una solicitud de revisión.

${ }^{33}$ Ibid, pár. 58.

${ }^{34}$ Ibid, pár. 35.

${ }^{35}$ Ibid, pár. 22. 


\section{Conclusiones}

A pesar de todos los esfuerzos realizados por El Salvador, a fin de presentar pruebas que demostraran la avulsión del Goascorán y por tanto, llenar las ausencias que la Sala en 1992 señaló en los párrafos 308 y 309, que hubieran podido haber hecho cambiar su decisión si se hubieran presentado en el momento oportuno, la Sala, en 2003 decidió que las pruebas aportadas en este momento no eran de naturaleza decisiva para el caso.

De lo que se desprende que, cada Estado que decide litigar ante el máximo tribunal de justicia internacional, debe poner todos sus esfuerzos a fin de no perder la oportunidad de probar sus derechos, mediante todos los tipos de pruebas que considere adecuadas, en el momento oportuno, debido a que después, ya es muy tarde.

El atentar contra la cosa juzgada, sigue siendo uno de los más grandes temores de todos los órganos de administración de justicia y ante dos males, siempre se escoge el menor.

\section{BIBLIOGRAFÍA}

- ${ }^{\circ}$ DIEZ-HOCHLEITNER; (REMIRO BROTONS-Director), Derecho Internacional, McGrow-Hill/Interamericana de España, Madrid, 1997.

- GUYOMAR, Geneviève. Commentaire du Règlement de la Court Internationale de Justisce, Pedone, Paris, 1983.

- ROSENNE, Shabtai. The law and practice of the International Court, Martinus Nijhoff, Dordrecht, Holanda, 2da Ed., 1985.

- ROSENNE, Shabtai. Procedure in the International Court, Martinus Nijhoff, La Haya, 1983.

- VERZIJL, JHM. The jurisprudence of the World Court. Vol. I, A.W. Sijthoff, Leyden, 1965.

Resúmenes de los fallos, opiniones consultivas y providencias de la Corte Internacional de Justicia 1948-1991, Naciones Unidas, Nueva York, 1992.

\section{Reportes de la Corte Internacional de Justicia}

- Application for revision and interpretation of the Judgment of 24 February 1982 in the case concerning the Continental Shelf (Tunisia/Libyan Arab Jamahiriya), Judgment of 10 December 1985, ICJ Reports 1985 p. 192. 
- Application for permission to intervene by the Government of Nicaragua, filed in the Registry of the Court on 17 November 1989.

- Land, Island and Maritime Frontier Dispute (El Salvador/Honduras) Application to Intervene, Judgment, ICJ Reports 1990, p. 92.

- Land, Island and Maritime Frontier Dispute (El Salvador/Honduras: Nicaragua intervening), Judgment of 11 September 1992, ICJ Reports 1992, p.351.

- Application for revision of the judgment of 11 July 1996 in the Case concerning application for the convention on the prevention and punishment of the crime of genocide (Bosnia and Herzegovina v. Yugoslavia), Preliminary Objections, (Yugoslavia v. Bosnia and Herzegovina), Judgment of 3 February 2003.

- Application for revision of the judgment of 11 September 1992 in the case concerning the Land, Island and Maritime frontier dispute (El Salvador/Honduras: Nicaragua intervening), (El Salvador v. Honduras) Jugdment of 18 December 2003. 